\title{
Fourier revisitado: um modelo simplificado para o efeito estufa
}

\author{
Fourier revisited: a simplified model for the greenhouse effect \\ Herman Fialho Fumiã ${ }^{1}$, Saulo Luis Lima da Silva ${ }^{*}[$ \\ ${ }^{1}$ Pesquisador autônomo, Divino, MG, Brasil. \\ ${ }^{2}$ Centro Federal de Educação Tecnológica de Minas Gerais, Nepomuceno, MG, Brasil.
}

\begin{abstract}
Recebido em 15 de março de 2021. Revisado em 11 de outubro de 2021. Aceito em 19 de dezembro de 2021.
Um dos assuntos mais discutidos no mundo atualmente são os efeitos das mudanças climáticas. Acredita-se que este será o tema de maior relevância no século XXI. No entanto, devido às complexidades inerentes aos sistemas climáticos, os modelos teóricos para se estudar tais fenômenos são muito complexos e exigem formação sólida e aprofundada na área. Neste trabalho, apresentamos um modelo simples e didático que, embora limitado, é capaz de mostrar como a atmosfera influi na regulação do clima no planeta. O modelo permite também vislumbrar a forma como os gases do efeito estufa alteram a transmissão da radiação no espectro do infravermelho através da atmosfera. Devido a seu forte apelo didático, interdisciplinar e atual, acreditamos que o trabalho possa ser muito interessante tanto para estudos em disciplinas de graduação quanto para aqueles que desejam se lançar nos estudos ligados a fenômenos atmosféricos e climáticos.
\end{abstract}

Palavras-chave: Aquecimento global, Efeito estufa, Mudanças climáticas.

\begin{abstract}
Climate change is one of the most debated issues today. Some researchers argue that this will be the most relevant topic in the 21st century. However, due to the complexities inherent to climatic systems, the theoretical models for studying such phenomena are very complex and require solid training in the area. In this work, we present a simple and didactic model that is able to show how the atmosphere influences the climate regulation on the planet. With this model, it is possible to have an idea of how greenhouse gases alter the transmission of radiation in the infrared spectrum through the atmosphere. Due to its didactic, interdisciplinary and current appeal, we believe that the work can be very interesting both for studies in undergraduate disciplines and for those who wish to launch themselves in studies related to atmospheric and climatic phenomena.
\end{abstract}

Keywords: Global warming, Greenhouse effect, Climate change.

\section{Introdução}

A história demonstra que nossa espécie vive em um movimento pendular com relação à perceção que tem sobre seu lugar no mundo. Nossa jornada começa como uma espécie tentando se equilibrar em apenas dois membros, mais lenta e fraca fisicamente que nossos primos mais próximos. A extinção não seria surpresa!

Ao que tudo indica, nossos cérebros maiores nos permitiram dominar o fogo e confeccionar artefatos complexos. Passamos de uma espécie vulnerável a uma espécie dominante. Domesticação de animais, revolução agrícola, invenção da escrita e formação de sociedades complexas e flexíveis foram nossos passos seguintes [1]. Olhando por esse prisma, não nos surpreende o fato de que as primeiras explicações modernas racionais como, por exemplo, a filosofia grega, nos tenha dado um lugar de destaque no Universo.

Com o Renascimento, por volta do século XV, o pêndulo movimenta-se em direção ao extremo oposto do

\footnotetext{
*Endereço de correspondência: saulolimasilva@yahoo.com.br
}

pensamento grego e somos forçados a deixar o centro do Universo e a ocupar a periferia de uma galáxia periférica, em um sistema dominado por uma estrela nada especial em que nossa presença nem sequer é percebida.

É nesse extremo do pêndulo, nessa posição onde nos vemos como insignificantes no Universo que o mundo tal como conhecemos pode estar perto do fim. Assistimos a cidades sendo engolidas pelo avanço do mar, massas de geleiras derretendo a uma taxa nunca antes registrada, temperatura média global aumentando e fenômenos naturais como furacões, tornados, maremotos cada vez mais devastadores se tornando corriqueiros [2]. A questão que se coloca é: essa espécie tão insignificante conhecida como homo sapiens pode ser a responsável por tamanha devastação?

Nas décadas de 1820, ainda nos primórdios de Revolução Industrial, Joseph Fourier (1768-1830) deu uma grande contribuição à resposta dessa questão. Fourier fez um balanço entre a quantidade de energia enviada a nosso planeta pelo Sol e quantidade de energia que a Terra reemite para o universo. De acordo com esse balanço, a temperatura da Terra deveria ser muito mais 
baixa do que é. Ele então especulou que a atmosfera deveria reter calor para manter sua temperatura, como um cobertor ou estufa. Fourier previu o efeito estufa, embora não tenha lhe dado esse nome $[3]^{1}$.

A intensidade do efeito estufa está diretamente relacionada a constituição química da atmosfera. Ao que tudo indica, a constituição atual de nossa atmosfera é produto da longa história evolutiva da vida na Terra, sendo que os microorganismos provavelmente determinaram a composição básica da atmosfera desde a origem da vida [4]. Dessa forma, a simbiose é tal que a composição química da atmosfera promove as condições para a vida e a própria vida regula a composição química da atmosfera [5].

O aumento da atividade humana pós-Revolução Industrial tem alterado a composição da atmosfera aumentando a concentração dos assim denominados gases do efeito estufa. O aumento da concentração desses gases está, de acordo com as evidências disponíveis, associado ao aquecimento do planeta [6, 86]2 Contudo, devido às complexidades inerentes aos sistemas climáticos, não podemos até o momento afirmar de forma precisa o quão rápido ou de quanto será esse aquecimento. Como um exemplo do tipo de previsão possível, os melhores modelos indicam que se a concentração de dióxido de carbono dobrar na atmosfera teremos um aumento da temperatura média terrestre entre $1,5^{\circ} \mathrm{C}$ e $4,5^{\circ} \mathrm{C}[8$.

Como podemos ver, embora tenha se passado quase dois séculos dos trabalhos de Fourier, a questão sobre a influência antrópica no clima global ainda é assunto de fronteira e de grande interesse na ciência e também na política. Tanto é que o prêmio Nobel de física de 2021 foi dado a cientistas que fizeram grandes contribuições nessa área 3

Neste trabalho, nosso objetivo é apresentar um modelo simples para o efeito estufa que se utiliza apenas de conceitos de física básica. A ideia é mostrar que mesmo sem usar os conceitos mais avançados das ciências atmosféricas é possível compreender a base sobre a qual se a assenta a afirmação de que os gases do efeito estufa podem exercer uma influência fundamental na regulação da temperatura do nosso planeta.

O trabalho está estruturado da seguinte forma: na Seção 2 apresentamos o modelo que propomos para estudar o problema. Na Seção 3 será apresentado os principais resultados que o modelo nos forneceu, nessa Seção também fazemos as discussões dos resultados obtidos. A Seção 4 é dedicada às conclusões do trabalho.

\footnotetext{
1 Em 1827 Fourier fez pequenas modificações em seu trabalho e o publicou como "Mémoire sur les températures du globe terrestre et des espaces planétaires" em Mém. Acad. Sci., 7. Em 1837 a Ebeneser Burgess publicou a tradução para o inglês do trabalho de 1824 na Amer. J. Sci., 32.

2 Segundo um relatório publicado na the Lancet, as mudanças climáticas são a maior ameaça à saúde no século XXI 7].

3 O prêmio Nobel de física de 2021 foi para o físico Giorgio Parisi, por seus trabalhos em sistemas complexos, e aos físicos Syukuro Manabe e Klaus Hasselmann pela proposta de um modelo físico do clima da terra.
}

\section{O Modelo}

Qualquer modelo físico pressupõem um conjunto de proposições (premissas) assumidas como verdadeiras. O modelo em questão assume as seguintes premissas:

1. O Sol é a única fonte de energia para os planetas;

2. A superfície dos planetas emite radiação como um corpo negro;

3. A atmosfera dos planetas constitui-se de uma camada de gases com uma temperatura uniforme;

4. A transferência de energia na atmosfera ocorre apenas por processos radiativos, ou seja, não existe transferência de energia por processos não radiativos como, por exemplo, o movimento de fluidos;

5. A atmosfera transmite a radiação solar de longos comprimentos de onda a uma taxa distinta da que transmite a radiação com comprimentos de ondas curtos.

\subsection{A energia solar que chega aos planetas}

A luminosidade solar $L_{\odot}$, estimada em $3,828 \times 10^{26} \mathrm{~W}$, é uma medida da taxa com a qual o Sol emite radiação eletromagnética. Sendo o Sol a única fonte de energia no modelo (premissa 1), é imperativo determinarmos uma expressão para a parcela de energia emitida pelo Sol que alcança um determinado planeta.

A energia emitida pelo Sol se propaga como uma frente de onda esférica espaço afora (Figura 1). A uma determinada distância $r$ do Sol e considerando um intervalo de tempo infinitesimal $\Delta t$, observamos que a energia emitida pelo Sol é igual a energia contida dentro da casca esférica de espessura infinitesimal $\Delta r=c \Delta t$. A energia total dessa casca é dada por:

$$
\Delta E=L_{\odot} \Delta t
$$

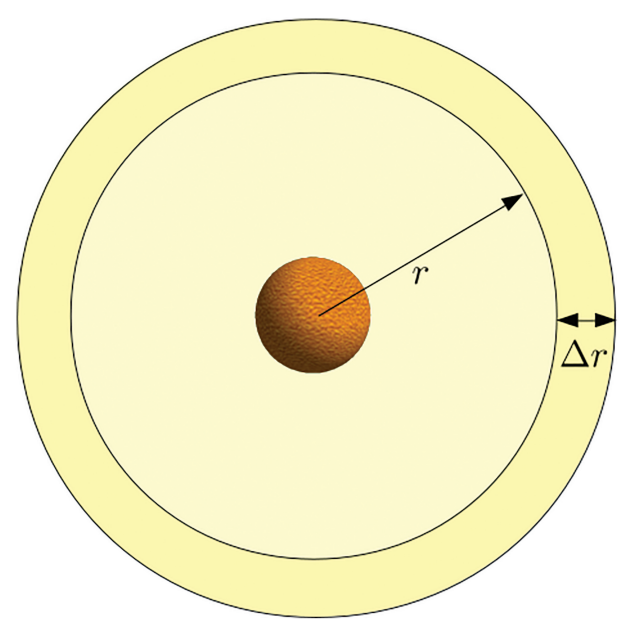

Figura 1: Representação esquemática, bidimensional, da propagação da energia emitida pelo sol, em forma de casca esférica, em um tempo $\Delta t$. 


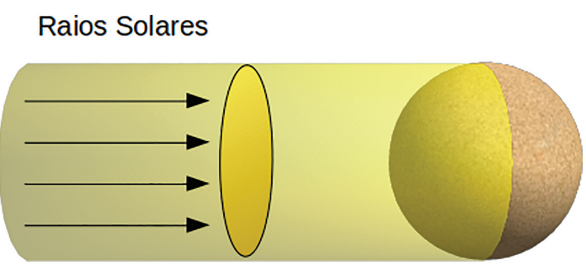

Figura 2: Figura ilustrativa da incidência dos raios solares em um planeta. Devido a grande distância até o Sol, os raios chegam praticamente paralelos e, portanto, contidos em um cilindro com um raio igual ao raio $r_{p}$ do planeta.

Por sua vez, a densidade de energia dessa casca é dada por:

$$
\sigma_{e}=\frac{L_{\odot} \Delta t}{4 \pi r^{2}} .
$$

A densidade de potência, denominada irradiância, é dada por:

$$
I=\frac{\sigma_{e}}{\Delta t}=\frac{L_{\odot}}{4 \pi r^{2}} .
$$

Devido às grandes distâncias que os planetas se encontram do Sol, os raios solares chegam essencialmente paralelos a eles. Dessa forma, a quantidade de energia que é interceptada por um determinado planeta está contida em um cilindro com área de seção reta igual a $\pi r_{p}^{2}$, em que $r_{p}$ é o raio do planeta em questão (Figura 2). Portanto, a energia solar total por unidade de tempo recebida pelo planeta é dada por:

$$
P_{p}=I \pi r_{p}^{2}=\frac{L_{\odot}}{4}\left(\frac{r_{p}}{r}\right)^{2},
$$

em que $r$ é a distância do planeta até o Sol.

\subsection{A temperatura dos planetas}

No modelo, existem duas camadas homogêneas: a atmosfera, que consiste em um uma camada de gases com uma temperatura constante $T_{a}$; e a superfície do planeta, que consiste em uma camada com uma temperatura constante $T_{s}$ (Figura 3).

Conforme apresentado na Figura 3 a potência de energia solar que chega a um determinado planeta é dada por $P_{p}$. Parte dessa energia é refletida diretamente para o espaço, tanto pela atmosfera quanto pela superfície do planeta 4 Essa potência refletida $P_{r}$ é dada por:

$$
P_{r}=\alpha P_{p}
$$

\footnotetext{
${ }^{4}$ Existe uma simplificação deliberada aqui ao unirmos a potência refletida pela atmosfera e pela superfície em uma única potência $P_{r}$. A alternativa mais realista é considerar a potência refletida pela atmosfera $P_{\text {ratm }}$ e depois a parte que é refletida pela superfície após ser trasmitida pela atmosfera $P_{r t s}$ separadamente. O modelo assim considerado produz equações para as temperaturas $T_{s}$ e $T_{a}$ homeomorfas às obtidas com o modelo apresentado. Contudo, esse modelo aumenta a complexidade do problema e, principalmente, insere dois parâmetros de difícil determinação: o albedo da atmosfera $\alpha_{a t m}$ e o da superfície $\alpha_{s}$.
}

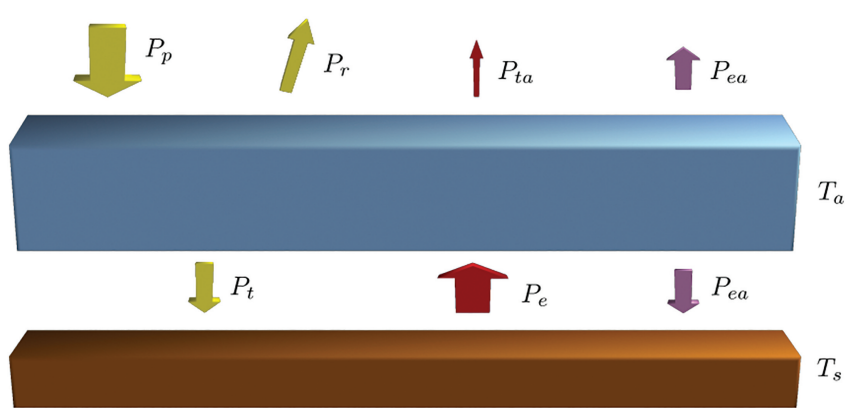

Figura 3: Modelo com atmosfera de uma camada. Em azul temos a camada correspondente à atmosfera e, em marrom, a camada que corresponde à superfície do planeta. A atmosfera e a superfície possuem temperaturas constantes dadas, respectivamente, por $T_{a}$ e $T_{s}$. A potência $P_{p}$ é aquela que chega até o planeta diretamente do Sol. $P_{r}$ é a potência refletida pela atmosfera e a superfície do planeta diretamente para o espaço. $P_{t}$ é a potência que é transmitida através da atmosfera e alcança a superfície do planeta. $P_{e}$ é a potência emitida pela superfície do planeta. $P_{t a}$ é a parcela da potência $P_{e}$ que consegue atravessar a atmosfera e escapar para o espaço. $P_{e a}$ é a potência emitida pela atmosfera. Observe que $P_{e a}$ é emitida tanto em direção ao espaço quanto em direção à superfície do planeta.

em que $\alpha$ é o coeficiente de reflexão, também conhecido como albedo.

Denominamos de $P_{0}$ a diferença entre a potência que chega ao planeta, $P_{p}$, e a aquela que é refletida para o espaço, $P_{r}$, ou seja:

$$
P_{0}=P_{p}-P_{r}=(1-\alpha) P_{p}
$$

A potência $P_{t}$ que é transmitida através da atmosfera e que, consequentemente, alcança a superfície, é dada por:

$$
P_{t}=t_{s} P_{0}
$$

em que $t_{s}$ é a transmissibilidade da atmosfera para a radiação solar.

Graças à premissa 2, podemos utilizar a Lei de StefanBoltzmann para obter a potência emitida pela superfície do planeta $P_{e}$ :

$$
P_{e}=4 \pi r_{p}^{2} \sigma T_{s}^{4}
$$

A potência $P_{t a}$ corresponde à parcela da potência emitida pela superfície $P_{e}$ que atravessa a atmosfera e escapa para o espaço. Ela é dada por:

$$
P_{t a}=t_{i} P_{e}=t_{i} 4 \pi r_{p}^{2} \sigma T_{s}^{4}
$$

em que $t_{i}$ é a transmissibilidade da atmosfera para a radiação emitida pela superfície.

A atmosfera não se comporta como um corpo negro. Portanto, podemos dizer que ela emite radiação a uma 
taxa dada por ${ }^{5}$

$$
P_{e a}=\varepsilon 4 \pi r_{p}^{2} \sigma T_{a}^{4},
$$

com a emissividade $\varepsilon<16$

A emissividade da atmosfera pode ser determinada pela Lei de Kirchhoff, a qual estabelece que a emissividade de um corpo em equilíbrio termodinâmico é necessariamente igual a sua absorvência. Como sabemos que a potência emitida pela superfície é em parte absorvida e em parte transmitida pela atmosfera, pelo Princípio de Conservação da Energia temos que:

$$
P_{e}=P_{\text {absorvido }}+P_{t a}
$$

e, $\log \mathrm{O}:$

$$
P_{\text {absorvido }}=P_{e}-P_{t a}=\left(1-t_{i}\right) 4 \pi r_{p}^{2} \sigma T_{s}^{4} .
$$

Portanto, como a absorvência é dada por $\left(1-t_{i}\right)$, a emissividade $\varepsilon$ também o é. Logo:

$$
P_{e a}=\left(1-t_{i}\right) 4 \pi r_{p}^{2} \sigma T_{a}^{4} .
$$

Como a transmissão de energia ocorre apenas por processos radiativos (premissa 4) e como o sistema encontrase em equilíbrio termodinâmico, podemos afirmar que:

$$
P_{e}=P_{t}+P_{e a}
$$

e

$$
P_{0}=P_{t a}+P_{e a}
$$

Agora, fazendo uso das Equações (9), (10), (14), (8) e (7), podemos substituir os termos $P_{0}, P_{t a}$ e $P_{e a}$ em 15) para obtermos as temperaturas $T_{s}$ e $T_{a}$. Feito isso, obtemos que

$$
T_{s}=\left(\frac{\left(1+t_{s}\right)(1-\alpha)}{\left(1+t_{i}\right)} \frac{L_{\odot}}{16 \pi \sigma r^{2}}\right)^{1 / 4},
$$

e

$$
T_{a}=\left(\frac{\left(1-t_{s} t_{i}\right)(1-\alpha)}{\left(1-t_{i}^{2}\right)} \frac{L_{\odot}}{16 \pi \sigma r^{2}}\right)^{1 / 4}
$$

\section{Resultados e Discussão}

\subsection{Temperatura de equilíbrio}

Ignorando a premissa 5 por um momento e supondo que $t_{i}=t_{s}=1$, temos uma atmosfera totalmente transparente. Com essa suposição, a expressão para a temperatura da superfície $T_{s}$ se reduz a:

$$
T_{s}=\left((1-\alpha) \frac{L_{\odot}}{16 \pi \sigma r^{2}}\right)^{1 / 4}
$$

\footnotetext{
$\overline{5}$ Observe que desprezamos a altura da atmosfera ao considerarmos $r_{p}$ como sendo o raio do planeta.

${ }^{6}$ A emissividade de um corpo negro é igual a 1 .
}

Tabela 1: Temperatura de equilíbrio $T_{e q}$, temperatura média real $\langle T\rangle$ e a diferença entre essas temperaturas $\Delta T$ para os planetas do sistema solar. Os valores para as distâncias médias dos planetas até o Sol $r$ e os seus albedos $\alpha$ foram obtidos de [10].

\begin{tabular}{l|c|c|c}
\hline Planeta & $T_{e q}\left({ }^{\circ} \mathrm{C}\right)$ & $\langle T\rangle\left({ }^{\circ} \mathrm{C}\right)$ & $\Delta T=\langle T\rangle-T_{e q}$ \\
\hline Mercúrio & 164 & 167 & 3 \\
Vênus & 231 & 464 & 233 \\
Terra & -19 & 15 & 34 \\
Marte & -63 & -65 & -2 \\
Júpiter & -163 & -110 & 43 \\
Saturno & -192 & -140 & 52 \\
Urano & -215 & -195 & 20 \\
Netuno & -227 & -200 & 27 \\
\hline
\end{tabular}

e, por sua vez, a temperatura da atmosfera $T_{a}$ se torna

$$
T_{a}=0
$$

A temperatura $T_{s}$ obtida dessa forma é denominada de temperatura planetária de equilíbrio $T_{e q}=T_{s}$.

Uma atmosfera totalmente transparente é equivalente a ausência de uma atmosfera. Dessa forma, a equação (18) nos fornece a temperatura que um planeta teria ${ }^{7}$ ou tem caso não possua uma atmosfera. Na Tabela 1 comparamos a temperatura de equilíbrio $T_{e q}$ para os planetas com a sua temperatura média real $\langle T\rangle$.

Da Tabela 1] observamos que os valores das temperaturas $T_{e q}$ e $\langle T\rangle$ diferem pouco para os planetas Mercúrio e Marte. Portanto, é de se esperar que esses planetas não possuam atmosferas consideráveis. De fato, por ser pequeno e próximo do Sol, Mercúrio possui uma atmosfera com uma pressão superficial de cerca de $10^{-12}$ bar [10] (aproximadamente $10^{-10} \%$ daquela da Terra), enquanto Marte possui uma atmosfera rarefeita com uma pressão atmosférica correspondendo a menos do que $1 \%$ daquela do nosso planeta [11]. A discordância acentuada entre as temperaturas de equilíbrio $T_{e q}$ e média $\langle T\rangle$ para os demais planetas sugere que desconsiderar a atmosfera nesses casos não é uma opção razoável.

\section{2. $\Delta T$ e o efeito estufa}

A diferença de temperatura $\Delta T=\langle T\rangle-T_{e q}$ é uma espécie de quantificação do efeito estufa e, portanto, útil para observarmos o papel da atmosfera na modificação da temperatura planetária. Entretanto, na maior parte das vezes, o valor calculado para $\Delta T$ não é uma boa estimativa de o quanto a temperatura de um planeta se modifica ao acrescentarmos uma atmosfera, é apenas uma grandeza correlacionada a essa modificação.

Ao calcularmos a temperatura de equilíbrio $T_{e q}$ na Tabela 1 utilizamos os albedos medidos para os planetas tal como eles são. Contudo, o albedo é uma grandeza que depende das características da superfície e também

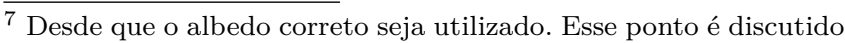
mais detalhadamente na Subseção 3.2
} 
da atmosfera do planeta. Não seria de se esperar que ele permanecesse o mesmo caso o planeta não tivesse atmosfera. Estima-se que uma Terra hipotética sem atmosfera, por exemplo, teria um albedo por volta de 0,14 [12]. Sendo assim, ao utilizarmos o albedo de 0,306 para determinarmos a temperatura de equilíbrio $T_{e q}$ não podemos afirmar que essa é a temperatura que a Terra teria caso não tivesse atmosfera, para isso deveríamos utilizar o valor de 0,14 .

Não existe problema algum em utilizarmos os valores dos albedos medidos $\$^{8}$ para calcularmos $T_{e q}$ e $\Delta T$ para os planetas, desde que não demos a interpretação errada para essas grandezas. Entretanto, esse é um erro comum mesmo na literatura especializada. A afirmação de que devido ao efeito estufa a temperatura da Terra é cerca de $33 \mathrm{~K}$ mais elevada do que seria caso ela não possuísse uma atmosfera ${ }^{9}$ é baseada em um cálculo que utiliza o albedo medido para a Terra com atmosfera. Utilizando o albedo teórico para a Terra sem atmosfera no cálculo de $T_{e q}$, obtemos que o aumento de temperatura devido ao efeito estufa está por volta de $20 \mathrm{~K}$, consideravelmente abaixo dos $33 \mathrm{~K}$ tão propalados.

\subsection{Presença de atmosfera}

A premissa 5 estabelece que os comprimentos de ondas curtos e longos são transmitidos a uma taxa distinta pela atmosfera. De fato, isso é o que poderíamos chamar de situação real.

$\mathrm{Na}$ Figura 4 observamos como a radiação é transmitida pela atmosfera terrestre para diferentes comprimentos de onda. A radiação solar, em vermelho, é composta predominantemente por comprimentos de ondas mais curtos, localizados na faixa do visível (pico por volta de $0,5 \mu \mathrm{m})$. Como vemos da figura, entre $70-75 \%$ dessa radiação atravessa atmosfera e atinge a superfície do planeta. Por outro lado, a radiação emitida pela superfície do planeta, em azul, corresponde a longos comprimentos de onda, situados na faixa do infravermelho (pico por volta de $10 \mu \mathrm{m}$ ). Da figura, vemos que entre $15-30 \%$ dessa radiação consegue atravessar a atmosfera rumo ao espaço.

Na Figura 5, utilizamos os parâmetro típicos da Terra para determinarmos a temperatura da superfície $T_{s}$. Utilizando os valores para $t_{s}$ e $t_{i}$ segundo [8], delimitamos uma região no gráfico para a temperatura $T_{s}$ dada pelo modelo. Essa região, delimitada pelas curvas correspondentes a $t_{s}=0,70$ e $t_{s}=0,75$ e pelas linhas verticais azuis correspondentes $t_{i}=0,15$ e $t_{i}=0,30$,

\footnotetext{
8 Principalmente, porque não é tarefa de pouca monta determinar os albedos teóricos correspondentes a todos os planetas hipotéticos sem atmosfera.

${ }^{9}$ Como exemplos dessa afirmação temos: "As a result, the mean global temperature of the Earth is about $33^{\circ} \mathrm{C}$ higher than it would be without the atmosphere..." ([8], na página 9); "The greenhouse effect is also noticeable on Titan and Earth, where the temperature is raised by $21 \mathrm{~K}$ and $33 \mathrm{~K}$, respectively, compared with their equilibrium temperatures."([10, na página 104).
}

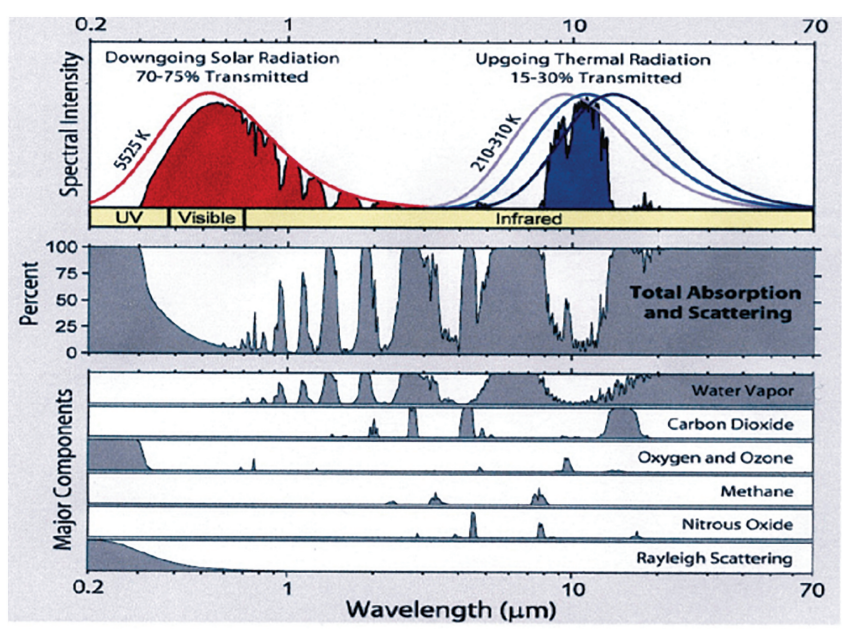

Figura 4: Radiação transmitida pela atmosfera terrestre. Retirado de 8 .

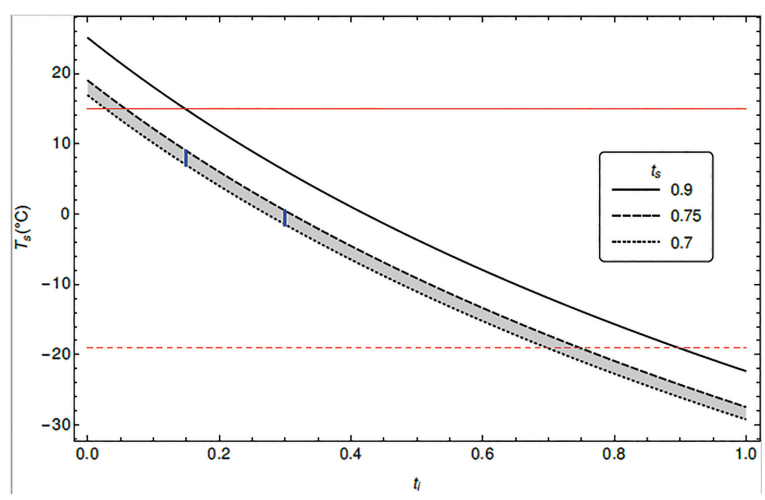

Figura 5: Temperatura da superfície $T_{s}$ para o planeta Terra em função de $t_{i}$ para três diferentes valores de $t_{s}$. A área delimitada pelas curvas correspondentes a $t_{s}=0,70$ e $t_{s}=0,75 \mathrm{e}$ pelas linhas verticais azuis corresponde a região com valores dos parâmetros $t_{i}$ e $t_{s}$ adequados para a Terra de acordo com [8]. A curva para $t_{s}=0,90$ é apresentada porque este é o valor utilizado em [9]. As linhas vermelhas correspondem a: temperatura média real $\langle T\rangle$ (linha superior) e a temperatura de equilíbrio $T_{e q}$ (linha inferior), ambas para a Terra.

está associada a temperaturas $T_{s}$ bem superiore ${ }^{10}$ que a temperatura de equilíbrio $T_{e q}$ para a Terra. Esse aumento da temperatura superficial do planeta devido a uma atmosfera que absorve mais radiação na faixa do infravermelho é o famigerado efeito estufa.

Embora a Figura 5 deixe claro o papel da atmosfera no aumento da temperatura da superfície $T_{s}$ do planeta, é notório que a faixa de valores obtidos para a temperatura $T_{s}$ está aquém do valor real médio para a temperatura terrestre $\langle T\rangle$. De fato, existem outros processos conhecidamente importantes para o balanço de

$\overline{10}$ Para $t_{s}=0,70$ e $t_{i}=0,30$, temos que $T_{s}=-1,49^{\circ} \mathrm{C}$ e, para $t_{s}=0,75$ e $t_{i}=0,15$, temos que $T_{s}=9,00^{\circ} \mathrm{C}$. Compare com a temperatura de equílibrio para a Terra $T_{e q}=-19^{\circ} \mathrm{C}$

$11 \mathrm{O}$ leitor atento deve ter observado que na Figura 5 uma curva para $t_{s}=0,90$ é apresentada. De fato, os valores de $t_{s}=0,90$ 
energia que foram deliberadamente ignorados no modelo. Para o planeta Terra, por exemplo, os mais importantes são os processos de condução e convecção de calor que ocorrem na atmosfera [9]. Portanto, um primeiro passo para um modelo mais refinado seria levantar a premissa 4. Para os planetas gigantes Júpiter, Saturno e Netuno, deve-se levar em conta também o calor interno produzido pelo planeta ${ }^{12}$ Nesse caso, a premissa 1 também deve ser levantada.

\subsection{O balanço de energia com e sem atmosfera}

Utilizando o planeta Terra como modelo e estabelecendo que a potência que chega até a Terra vinda do Sol $P_{p}$ é igual a 100 un., estudamos os fluxos de potência na atmosfera e as temperaturas $T_{a}$ e $T_{s}$ para três situações: (1) Uma atmosfera totalmente transparente; (2) uma atmosfera que não diferencia radiações com comprimentos de ondas distintos; (3) uma atmosfera real.

A Figura 6 apresenta os fluxos de potência e as temperaturas para a atmosfera totalmente transparente $\left(t_{s}=t_{i}=1,0\right)$. Como já citado, esse caso é, com as devidas ressalvas, equivalente a ausência de uma atmosfera. Como seria de se esperar, a temperatura de uma atmosfera que não absorve energia alguma é igual a $T_{a}=0 \mathrm{~K}$. Por sua vez, a temperatura da superfície do planeta $T_{s}$ é igual a temperatura de equilíbrio $T_{s}=$ $T_{e q}=-19^{\circ} \mathrm{C}$.

Observe que a potência que chega até a superfície do planeta é dada por $P_{t}=70 \mathrm{un}$., que é igual à potência que é emitida por essa superfície de volta para o espaço;

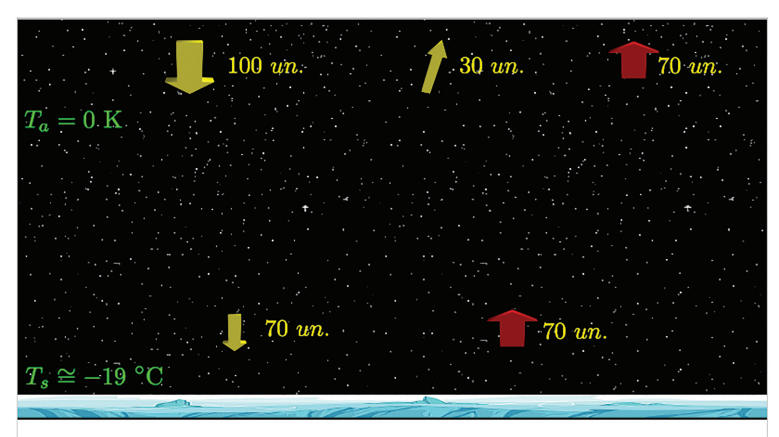

Figura 6: Balanço de energia para uma atmosfera totalmente transparente $\left(t_{s}=t_{i}=1,0\right)$. A setas amarelas representam as potências que chegam diretamente do Sol, atravessam a atmosfera ou são diretamente refletidas de volta para o espaço. As setas vermelhas representam as potências emitidas pela superfície ou que a partir dela atravessam a atmosfera.

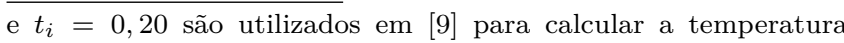
$T_{s}$, o que retorna um valor muito próximo do real, a saber, $13^{\circ} \mathrm{C}$. Contudo, como a fonte dos dados não é deixada clara e o autor utiliza a expressão "... Taking rough values for the Earth's atmosphere to be...", essa parece ser uma escolha mais didática do que precisa.

12 Estranhamente, Urano não produz muito calor interno

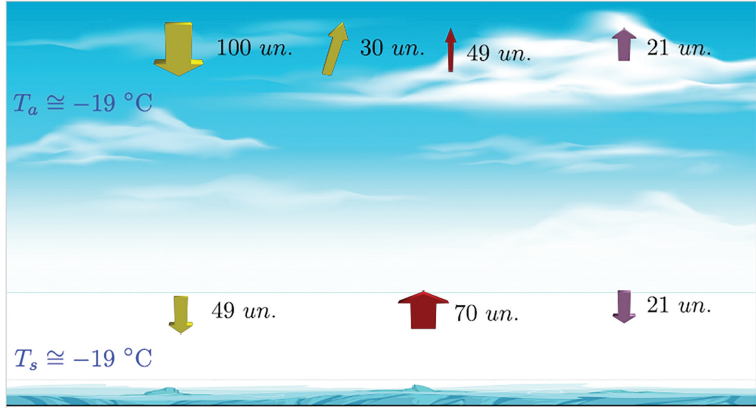

Figura 7: Balanço de energia com uma atmosfera que não discrimina entre comprimentos de ondas curtos ou longos $\left(t_{s}=\right.$ $\left.t_{i}=0,70\right)$. A setas amarelas representam as potências que chegam diretamente do Sol, atravessam a atmosfera ou são diretamente refletidas de volta para o espaço. As setas vermelhas representam as potências emitidas pela superfície ou que a partir dela atravessam a atmosfera. As setas roxas representam as potências emitidas pela atmosfera para o espaço e para a superfície.

condizente com uma atmosfera que não absorve ou emite radiação.

Na Figura 7, temos a situação para uma atmosfera que absorve energia em todos os comprimentos de onda de forma uniforme. Dessa maneira, tanto faz se a radiação incidente está na faixa do visível ou do infravermelho; ela será transmitida pela atmosfera na mesma proporção.

Como a atmosfera absorve energia, a potência $P_{t}$ que atinge a superfície diretamente é menor do que na situação anterior, sendo de 49 un. Entretanto, como a atmosfera emite de volta para a superfície uma potência $P_{e a}=21$ un., a potência total que chega até a superfície continua sendo de 70 un.. Isso explica o porquê da temperatura da superfície $T_{s}$ continuar sendo a mesma que aquela da temperatura de equilíbrio $T_{e q}$.

Distintamente da situação anterior, agora, a atmosfera possui uma temperatura não-nula, já que ela absorve energia. Além disso, a atmosfera encontra-se em equilíbrio térmico com a superfície; consequência do fato da atmosfera transmitir a radiação de forma uniforme.

Na Figura 8, temos o caso da atmosfera que transmite de forma distinta a radiação na faixa do infravermelho e do visível. A potência $P_{t}$ que chega à superfície diretamente é a mesma que na situação anterior $\left(P_{t}=49\right.$ un.). Todavia, a potência total que chega à superfície é bem maior (99 un). O aumento na potência total deve-se ao aumento na potência $P_{e a}$ emitida pela atmosfera $\left(P_{e a} \cong 50\right.$ un. $)$. Como a superfície do planeta absorve toda a energia incidente sobre ela, isso explica o aumento da temperatura da superfície do planeta $T_{s}$ em relação aos casos anteriores.

\subsection{A influência dos gases do efeito estufa}

Os principais gases do efeito estufa são o vapor d'água $\left(\mathrm{H}_{2} \mathrm{O}\right)$, dióxido de carbono $\left(\mathrm{CO}_{2}\right)$, metano $\left(\mathrm{CH}_{4}\right)$, óxido 


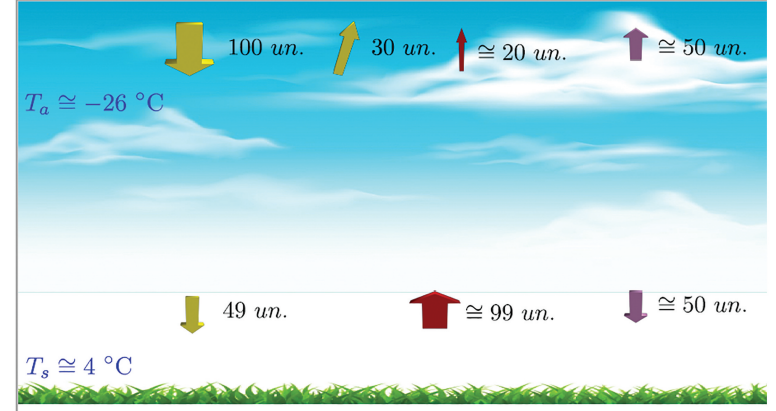

Figura 8: Balanço de energia com uma atmosfera "real" $\left(t_{s}=\right.$ 0,70 e $\left.t_{i}=0,20\right)$. A setas amarelas representam as potências que chegam diretamente do Sol, atravessam a atmosfera ou são diretamente refletidas de volta para o espaço. As setas vermelhas representam as potências emitidas pela superfície ou que a partir dela atravessam a atmosfera. As setas roxas representam as potências emitidas pela atmosfera para o espaço e para a superfície.

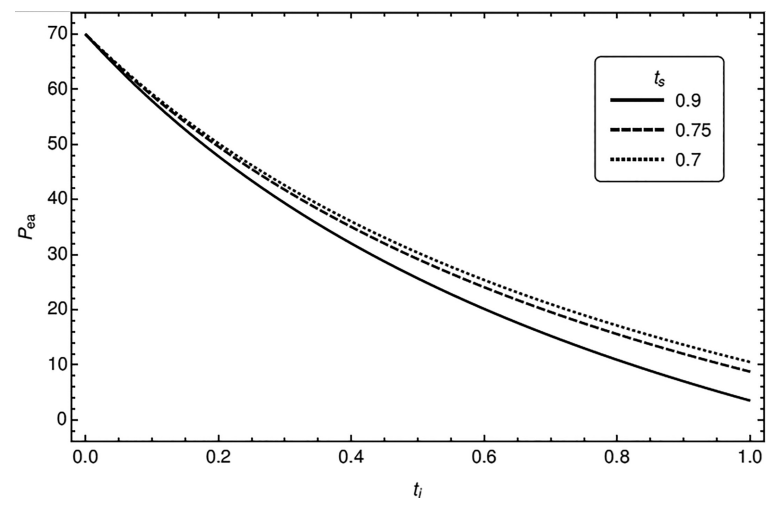

Figura 9: Potência emitida pela atmosfera em função de $t_{i}$ para diferentes valores de $t_{s}$. A diminuição de $t_{i}$ está relacionada a um aumento na concentração de gases do efeito estufa.

nitroso $\left(\mathrm{N}_{2} \mathrm{O}\right)$, ozônio $\left(\mathrm{O}_{3}\right)$, e os clorofluorcarbonetos (CFCs) [13].

Podemos definir os gases do efeito estufa como aqueles que emitem e absorvem a radiação na faixa do infravermelho, mas que não absorvem significativamente a radiação solar na faixa do visível [9]. Portanto, no modelo, variações nas concentrações de gases do efeito estufa na atmosfera estão diretamente relacionadas a alterações na transmissibilidade $t_{i}$. Dessa maneira, espera-se que uma maior concentração desses gases aumente a absorção na faixa do infravermelho, o que é o mesmo que dizer que haverá uma diminuição da transmissão dessa radiação ( $t_{i}$ diminui).

Como podemos ver da Figura 9, a potência emitida pela atmosfera terrestre $P_{e a}$ sempre aumenta quando a transmissibilidade $t_{i}$ diminui. Como o aumento na potência emitida pela atmosfera terrestre $P_{e a}$ significa mais radiação chegando à superfície, esperamos que a diminuição de $t_{i}$ também implique em um aumento na

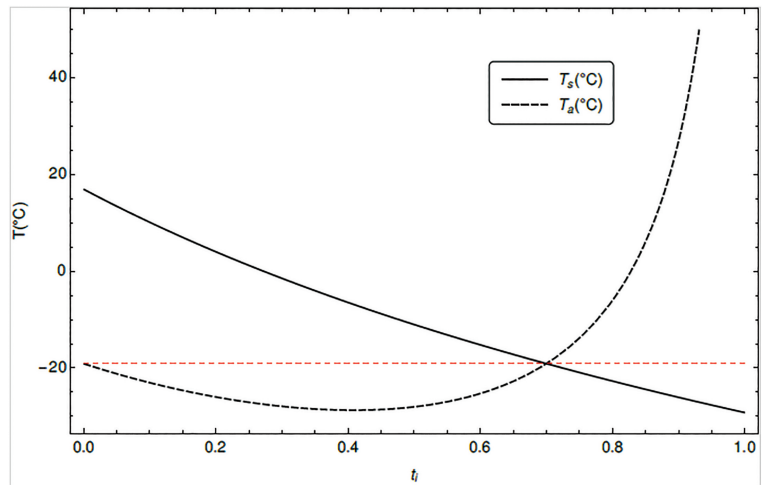

Figura 10: Temperatura da superfície $T_{s}$ e da atmosfera $T_{a}$ terrestre em função de $t_{i}\left(t_{s}=0,7\right)$. Observe que a temperatura $T_{s}$ sempre aumenta com a diminuição de $t_{i}$, enquanto a temperatura $T_{a}$ exibe comportamento consideravelmente mais complexo. A linha tracejada vermelha corresponde a temperatura de equilíbrio $T_{e q}$.

temperatura da superfície $T_{s}$. De fato, isso é o que observamos (Figura 10).

\section{Conclusão}

Os modelos para o clima que se prestam a projeções sobre o aumento da temperatura terrestre no tempo em diversos cenários de emissão de gases do efeito estufa são muito complexos e, portanto, mais restritos aos especialistas. Contudo, a "essência" de como a atmosfera influi na temperatura do planeta pode ser compreendida através de modelos simples, como o que apresentamos neste trabalho. $\mathrm{O}$ modelo apresentado também permite vislumbrar a forma como os gases do efeito estufa alteram a transmissão da radiação no espectro do infravermelho através da atmosfera e como isso pode estar associado ao aquecimento global.

Por último, acreditamos que o modelo apresentado seja útil, devido a sua simplicidade, como uma introdução para aqueles que desejam se lançar na seara de assunto tão importante quanto o aquecimento global. Acreditamos que nisso resida a sua força didática.

\section{Referências}

[1] Y.N. Harari, Sapiens (L\&PM, Porto Alegre, 2018).

[2] V. Thomas e R. López, Asian Devel. Bank Econ. Work. Pap. Ser. 466, 1 (2015).

[3] J. Fourier, Ann. Chim. Phys. 27, 136 (1824).

[4] J.F. Kasting e J.L. Siefert, Science 296, 1066 (2002).

[5] A.L. Junges, V.Y.D. Santos, N.T. Massoni e F.A. Santos, Exper. Ens. Ciências 13, 126 (2018).

[6] R. Neukom, N. Steiger, J.J. Gómez-Navarro, J. Wang e J.P. Werner, Nature 571, 550 (2019).

[7] N. Watts, M. Amann, N. Arnell, S. Ayeb-Karlsson, K. Belesova, H. Berry et al, The Lancet 392, 2479 (2018).

[8] G. Alimonti, EPJ Web of Conferences 189, 00003 (2018). 
[9] D.V. Andrews, An introduction to atmospheric physics (Cambridge University Press, Cambridge, 2010), v.1, p.7.

[10] J.J. Lissauer e I. De Pater, Fundamental planetary science: physics, chemistry and habitability (Cambridge University Press, Cambridge, 2013), v.1, p.522.

[11] R.M. Haberle, em: Encyclopedia of Atmospheric Sciences, editado por G.R. North, J. Pyle e F. Zhang (Academic Press, Cambridge, 2015), $2^{\mathrm{a}}$ ed.

[12] X. Zeng, Eos Trans 91, 134 (2010).

[13] P.R. Anderson, C.M. Júnior e M.J.S. Júnior, Rev. Bras. Ensino Fís. 43, e20200355 (2021). 